\title{
Instrumentos para develar indicadores de riesgo de la discalculia en estudiantes de Educación Primaria
}

\author{
Instruments to reveal risk indicators of dyscalculia in Primary Education students
}

\author{
Carlos José Martínez Aguirre
}

crlosmrtnz@gmail.com

Código ORCID: 0000-0002-9877-9184

Ministerio del Poder Popular para la Educación, Venezuela

Recibido: abril 2020 / Arbitrado: mayo 2020 / Publicado: julio 2020

RESUMEN

La presente investigación consiguió diseñar un instrumento para develar los indicadores de riesgo de la dificultad en el aprendizaje de la matemática (Discalculia) en los estudiantes de educación primaria. Dicho estudio quedó conformado por veinticuatro docentes de aula de la U.E. "Juan Antonio Michelena", donde la muestra fue $30 \%$, equivalente a siete docentes. Seguidamente, durante la recolección de los datos se aplicó una encuesta constituida por cincuenta ítems dicotómicos cuyas alternativas de respuestas fueron SI o NO, el mismo fue validado por cinco expertos. Luego, de aplicar dicho instrumento se obtuvo a través del coeficiente de KuderRichardson, un índice confiable de 0.8406 siendo considerado muy alto. De allí; se analizaron los datos arrojados por la encuesta y entre otras conclusiones se observó la necesidad de proveer al docente de la información necesaria acerca de los indicadores de riesgo de las Dificultades específicas en el Aprendizaje de la Matemática (Discalculia).

Palabras clave:

Instrumentos; indicadores de riesgo; discalculia

\begin{abstract}
The present investigation managed to design an instrument to reveal risk indicators of difficulty in learning mathematics (Dyscalculia) in primary school students. This study was made up of twenty-four classroom teachers from the U.E. "Juan Antonio Michelena", where the sample was $30 \%$, equivalent to seven teachers. Next, during data collection, a survey was applied consisting of fifty dichotomous items whose alternative answers were YES or NO, it was validated by five experts. After applying this instrument, a reliable index of 0.8406 was obtained through the Kuder-Richardson coefficient, which is considered very high. Thence; The data from the survey were analyzed and, among other conclusions, the need to provide the teacher with the necessary information about the risk indicators of the specific Difficulties in Learning Mathematics (Dyscalculia) was observed.
\end{abstract}

Keywords:

Instruments; risk indicators; dyscalculia 


\section{INTRODUCCIÓN}

La educación es un hecho social, y en primera instancia busca proveer al ser humano de los medios necesarios para enfrentar con éxito la vida y lograr su pleno desarrollo. La sociedad actual necesita una educación de calidad, dentro de un proceso continuo, total e integral con el fin de crear escenarios de formación holística. En este sentido la Ley Orgánica de Educación (2009) (LOE) plantea que el fin de la educación es "desarrollar el potencial creativo de cada ser humano para el pleno ejercicio de su personalidad y ciudadanía..." (art. 15, núm. 1). Esta meta sólo es posible si las estrategias, recursos y métodos implementados en el aula de clase explotan el máximo potencial de cada estudiante según sus capacidades, ofreciéndoles así, las herramientas necesarias para el logro de sus metas, y desde la educación; construir una sociedad armónica, coherente y honesta, cuyo resultado será un país más productivo y educado. Según Lake (2015) citado en Unesco (2017):

La educación es la clave para dar a cada niño una vida mejor y constituye el cimiento de todas las sociedades sólidas. Pero todavía hay muchos niños que se están quedando rezagados. Para cumplir nuestras metas de desarrollo, necesitamos que todos los niños vayan a la escuela y adquieran conocimientos. (p.12)

En este sentido, la Unesco en su Declaración de Incheon, Educación 2030, promueve el "Garantizar una educación inclusiva y equitativa de calidad y proponer oportunidades de aprendizaje permanente para todos" (p.1). Como organismo regente educativo a nivel mundial, también plantea dentro de sus tareas los objetivos de desarrollo sustentable hacia el año 2030, entre ellas se encuentra la meta 4.6 con la premisa de, "garantizar que todos los jóvenes y al menos una proporción sustancial de los adultos, tanto hombres como mujeres, tengan competencias de lectura, escritura y aritmética" (p.20). Sin embargo, en lo que concierne a la matemática, se caracteriza por ser una de las asignaturas menos populares entre adultos, jóvenes y niños.

En concordancia con la Unesco, en Venezuela la LOE (2009) en su artículo 6, numeral 1 decreta lo siguiente: "El derecho pleno a una educación integral, permanente, continua y de calidad para todos y todas con equidad de género en igualdad de condiciones y oportunidades, derechos y deberes." Por esta razón se puede decir que en Venezuela todos tienen libre acceso al sistema educativo, sin importar su condición. Es decir, la educación en Venezuela es inclusiva y además, garantiza "El acceso al Sistema Educativo a personas con necesidades educativas especiales $\mathrm{O}$ con discapacidad, mediante la creación de condiciones y oportunidades...", demostrando así estar en sincronía con los objetivos propuestos por la Unesco.

En relación al literal "c" de la misma ley previamente citada, refiere lo previsto para las Personas con Discapacidad (2007) (LPD), la cual expresa en su artículo 16, "Toda persona con discapacidad tiene derecho a asistir a una institución o centro educativo 
para obtener educación, formación o capacitación..." del mismo modo, tomando la definición de persona con discapacidad, en el artículo 5 explica lo siguiente:

Se entiende por discapacidad la condición compleja del ser humano constituida por factores biopsicosociales, que evidencia una disminución o supresión temporal o permanente, de alguna de sus capacidades sensoriales, motrices o intelectuales que puede manifestarse en ausencias, anomalías, defectos, pérdidas o dificultades para percibir, desplazarse sin apoyo, ver u oír, comunicarse con otros, o integrarse a las actividades de educación o trabajo, en la familia con la comunidad, que limitan el ejercicio de derechos, la participación social y el disfrute de una buena calidad de vida, o impiden la participación activa de las personas en las actividades de la vida familiar y social, sin que ello implique necesariamente incapacidad 0 inhabilidad para insertarse socialmente. (LPD, 2007)

En este contexto, las Unidades Educativas Estadales y Nacionales, deberían contar con un personal especialista como los psicopedagogos, cuya tarea sea la de diagnosticar e intervenir ante la existencia de alguna dificultad o necesidad presente en los estudiantes, a fin de proporcionarle al docente el apoyo preciso y atender de manera eficaz el contenido curricular, fomentando así, una educación inclusiva, equitativa y de calidad, puesto que lo exige el marco legal venezolano. Sin embargo, a la hora de atender las necesidades específicas en el aprendizaje del niño, en muchos casos el docente no posee las herramientas para contribuir con el diagnóstico de dicha dificultad. En este sentido se hace necesario el diseño continuo de estas herramientas, así como también la inclusión del docente en el proceso de diagnóstico que, como lo señala Fiuza y Fernández (2014):

Los maestros han de estar
atentos a los indicios de
aquellas conductas que
puedan ser indicadoras de
riesgo para, desde un
enfoque multidisciplinario,
colaborar activamente en
crear ambientes educativos en
los que tanto las dificultades
como los trastornos se
minimicen lo más posible y
todo el alumnado pueda
realizar aportaciones a la
comunidad, constituyéndose
como un sujeto que tiene
algo valioso que ofrecer a la
sociedad. (p. 15)

Así mismo, con frecuencia las clases de matemática consisten en explicaciones impartidas por el docente sobre los temas de un contenido programático, mientras que los estudiantes se limitan básicamente a escuchar, tomar apuntes y luego estudiar de memoria los procedimientos para los exámenes. $Y$ es allí donde ocurre que, en algunos casos el docente no alcanza a detectar las dificultades presentes en sus estudiantes, tal vez porque no posee las herramientas para interpretar los síntomas o indicadores reflejados por el estudiante, lo cual le impide actuar de manera eficaz ante 
la necesidad requerida. Del mismo modo, un gran número de factores hacen que esta práctica se perpetúe en el tiempo e imposibilite a los docentes en la implementación de nuevas estrategias que contribuyan a mejorar el rendimiento académico en los estudiantes que presentan dificultades en el aprendizaje de la matemática.

Dichas dificultades deberían ser diagnosticadas por un equipo de expertos, donde el docente, como primer eslabón del proceso, deberá estar atento a esos indicadores que puedan revelar dicha dificultad. Esta información es esencial en la evaluación del estudiante. Además, la ausencia de Psicopedagogos y orientadores dentro de los planteles para evaluar estos casos, y cuya intervención permitiría la identificación de las dificultades en el aprendizaje en el estudiante, el diseño de las estrategias que requiere el estudiante para aprender y el logro de los objetivos planificados por el docente, hace necesario que el profesor participe activamente en la detección temprana de cualquier dificultad en el aprendizaje de la matemática.

De esta manera es posible definir las Dificultades de Aprendizaje (DA), según Kirk (1963), quien sostiene que las miasmas se refieren a aquellos niños en edad escolar cuyo coeficiente intelectual a pesar de estar dentro de los límites normales, suelen presentar problemas al aprender ciertos aspectos más específicos. Esta dificultad de aprendizaje, generalmente se encuentra en el área de Matemática, y se le conoce como Discalculia. La Asociación Americana de Psiquiatría (2000) (DSM-IV-TR) la define como "una capacidad aritmética que se sitúa sustancialmente por debajo de la esperada en individuos de edad cronológica, coeficiente de inteligencia y escolaridad concordes con la edad, medida mediante pruebas normalizadas de cálculo o razonamiento matemático administradas individualmente" (p.52). Los estudiantes con esta dificultad de aprendizaje tienen problemas en muchos aspectos de la matemática y a menudo no comprenden las cantidades o conceptos como: "mayor que" o "menor que", o puede que no entiendan que el número " 6 " es igual a la palabra "seis".

Por otra parte, el estudio de la matemática no es nada sencillo, más bien, puede llegar a ser un proceso muy complejo, y requiere de ciertas habilidades cognitivas claves para lograr alcanzar el conocimiento matemático, González y Pienda (1998, citado en Coronado, 2012) afirman que "La matemática es a su vez uno de los conocimientos más necesarios de las sociedades modernas, también uno de los más difíciles de aprender" (p.7). Si bien es cierto; en el estudiante estas habilidades no se adquieren de manera innata, se necesita que en las primeras etapas de educación primaria se reciba una adecuada formación acorde a su edad cronológica y capacidad cognitiva.

En relación a esto, las cifras del Instituto de Evaluación del Ministerio de Educación Español indican que durante el 2010, el 22\% de los estudiantes en España sufrían de algún trastorno del aprendizaje; en comparación a otros países de Europa: Suecia, Francia, Austria, Portugal e Italia cuya incidencia fue de $21 \%$ y $25 \%$. Del mismo modo en los Estados Unidos de Norteamérica, se observó entre el año 2015 
y 2016 con respecto a las DA, que un 39\% de los estudiantes de educación pública fueron diagnosticados con las DA específicas de Dislexia (lectura), Digrafía (escritura) y Discalculia (matemática). Aunque existe un gran número de estudiantes sin diagnóstico, esta cifra podría aumentar si no se atiende de manera eficaz y oportuna.

Ahora bien, luego de ver estas cifras a nivel internacional donde las Dificultades del Aprendizaje (DA) son tan comunes y considerando la falta de datos oficiales publicados por las instituciones nacionales, como el Ministerio del Poder Popular para la Educación (MPPE), el Instituto Nacional de Estadísticas (INE) y la Comisión Nacional para las Personas con Discapacidad (CONAPDIS), se puede presumir que estos datos son similares dentro la población estudiantil Venezolana, por ello se hace necesario que el docente, como especialista de la educación conozca las dificultades de aprendizaje, especialmente de aquellas referentes a la matemática.

Si bien es cierto, no existen cifras oficiales $y$ estos datos no deben parecer menos importantes, porque el porcentaje de estudiantes detectados es bastante significativo en otros países pese a que poseen programas e instrumentos para el diagnóstico de las Dificultades de Aprendizaje y donde se elaboran planes de intervención temprana. Sin embargo, aún no han sido diagnosticados todos los casos. Mientras tanto, en Venezuela estas probabilidades deben ser objeto de estudio, evaluación, y diagnóstico para ocuparnos en atender este escenario en las aulas de clase reglares, pues no se conoce la cantidad de estudiantes que pueden sufrir este tipo de Dificultades Especificas de Aprendizaje los cuales deben ser identificados de manera oportuna para poder superarlo.

Esta fue la razón que dio paso a la realización de la presente investigación ya que contempla que la falta de conocimiento de algunos docentes sobre las Dificultades Especificas de Aprendizaje en Matemática es un problema que amerita ser investigado. De este modo se puede diseñar un instrumento que le permita al docente descubrir los indicadores de riesgo que se presentan en los estudiantes y con ello descartar si estas dificultades pueden estar afectando el rendimiento académico en la asignatura de Matemática.

En virtud de los planteamientos expuestos, surge la principal interrogante de la investigación: ¿Que instrumento se puede diseñar para permitir al docente de aula regular develar los indicadores de riesgo de las Dificultades Específicas de Aprendizaje en Matemática en sus estudiantes? Para responder a dicha pregunta de investigación se planteó el siguiente objetivo general: Diseñar instrumentos para develar los indicadores de riesgo de las dificultades específicas del aprendizaje de la matemática (Discalculia) en estudiantes de educación primaria.

Para alcanzar este objetivo final se formularon los siguientes objetivos específicos: 1. Diagnosticar el conocimiento que poseen los docentes de educación primaria sobre las Dificultades Específicas del Aprendizaje de la Matemática. 2. Estudiar la factibilidad de la creación de instrumento para develar los indicadores de riesgo de las Dificultades Específicas del Aprendizaje de la 
Matemática en estudiantes de educación primaria. 3. Diseñar el instrumento que permita develar los indicadores de riesgo de las dificultades específicas del aprendizaje de la matemática (Discalculia).

En función de lo anteriormente descrito, a continuación, se presentan los antecedentes relacionados con el objeto de estudio y por su contenido se ajustan a los objetivos con la finalidad de alcanzar el propósito de la presente investigación:

El primero está en Llumitaxi y Vera (2017), cuyo objetivo fue determinar los índices de Discalculia en el aprendizaje de las matemáticas de los niños del 4to. Año de educación general básica. Concluyó que el aprendizaje de las matemáticas requiere de una práctica constante para fortalecer habilidades y destrezas en esta área. Cuando no se habitúa la ejercitación de las nociones básicas y el razonamiento lógico, los niños están propensos a adquirir una Discalculia Ideognóstica u operacional, las cuales impiden al niño realizar operaciones mentales y relacionarlas con la escritura. Al mismo tiempo, 56\% de los niños presentan dificultades al ubicar los números que van antes o después dentro de una secuencia. Al mismo tiempo, $50 \%$ de los estudiantes no lograron resolver de una manera ordenada y rápida las multiplicaciones de 1 y 2 cifras, debido a su dificultad para ubicar los números en las cifras, además no saben las tablas de multiplicar. Por último, a pesar de esto los docentes afirman que realizan ejercicios diariamente lo que deja en evidencia la ineficacia de las estrategias aplicadas y estas no ayudan a los estudiantes a fortalecer las destrezas $y$ habilidades matemáticas.
En segundo lugar, se apreció el trabajo elaborado por, Méndez y Vivanco (2016). Dentro de sus objetivos estuvo determinar las afectaciones producidas por la Discalculia en los niños de 8 años de edad, en el caso particular del niño Carlos Masa Romero, el cual permitió el diagnóstico de Discalculia, evidenciando la existencia de este trastorno en el aprendizaje de matemática, ya que demostró no poder resolver un conjunto de operaciones matemáticas. Este estudio también permitió identificar algunas causas y consecuencias de la Discalculia en los niños de 8 años y su afectación en el proceso de desarrollo del pensamiento lógico, pues se encuentra por debajo de los conocimientos de la media de los demás compañeros del aula. Con estos resultados se pudo evidenciar la necesidad de un compromiso tripartito entre los padres, la maestra y el psicólogo educativo. Al finalizar, los autores declaran que no existe una Guía Didáctica para tratar la Discalculia, tampoco se realizan actividades innovadoras para disminuir con ellas los problemas de Discalculia y mejorar el proceso de aprendizaje en el área de matemática.

Así mismo, Oña (2016), identifica el ciclo de Kolb, como la estrategia metodológica utilizada para enseñar la matemática, donde en el proceso de aprendizaje existen 4 capacidades diferentes: la capacidad de experiencia concreta, la capacidad de observación reflexiva, la capacidad de conceptualización abstracta y por último la capacidad de experiencia activa. Este autor menciona que la dificultad de aquellos niños/as que poseen este problema de aprendizaje al momento de desenvolverse en 
el aula con niños de aprendizaje normal les causa pocos inconvenientes. Así mismo afirma que existen lineamientos en el Ministerio de Educación de Ecuador sobre las adaptaciones curriculares realizadas por los mismos docentes, estas sirven de guía en el proceso de enseñanza. La metodología, técnicas y estrategias son adaptadas a la necesidad del estudiante por cuanto son guiados por el Departamento de Consejería Estudiantil, el cual proporciona la información respectiva y lleva a cabo la inclusión educativa establecidas en el acuerdo ministerial.

También Sánchez (2014) presentó una investigación cuyo objetivo fue Establecer estrategias educativas tecnológicas para ayudar a los niños con Discalculia de primer año educación básica mediante la elaboración de recursos didácticos con herramientas multimedia. Las conclusiones preliminares fueron en primer lugar, la Discalculia en los niños puede ser tratada, y los padres también desempeñan un papel importante; en segundo lugar, el utilizar material multimedia didáctico actúa como un motivador que genera en los niños el deseo de aprender; en tercer lugar, el uso de estrategias metodológicas adecuadas a las necesidades de aprendizaje de cada niño permite desarrollar sus capacidades y habilidades; y por último recomienda proporcionar al docente recursos para desarrollar sus actividades pedagógicas más motivadoras y dinámicas.

Cerrando el recuento de los antecedentes está Santamaría (2014) cuyo trabajo tuvo el objetivo de estudiar la evaluación en la matemática de la educación primaria, donde se hizo un repaso de los grandes pensadores matemáticos, a lo largo de la historia, para sacar lo positivo de cada uno de ellos. Concluye que, a pesar de la gran variedad de técnicas y recursos para evaluar, siempre se recurre a las mismas técnicas tradicionales y la evaluación escrita que no garantiza verazmente el conocimiento de los estudiantes, argumentando que cualquiera puede tener un mal día y por tanto esos resultados no pueden ser representativos ni el sustento de una nota o valoración. Del mismo modo, se debe involucrar a los estudiantes como sujetos activos del proceso, desarrollando una perspectiva crítica, reflexiva y autocritica en su proceso de aprendizaje, afirmando con esto que, si los estudiantes logran desarrollar estas perspectivas en el momento de realizar y pensar en sus tareas, estos serán competentes a la hora de ponerlas en práctica en su vida cotidiana.

En resumen, dentro de los antecedentes descritos, además de detectar las dificultades de los estudiantes a través de pruebas no formales y diagnósticas, se obtuvieron datos estadísticos importantes donde se revela el alto porcentaje de educandos con ciertas dificultades, las cuales son indicadores claros de la variable objeto de estudio. De igual modo, establecen los factores que inciden para develar la presencia de Discalculia en los escolares y proporcionar algunos de los indicadores a tener en cuenta para identificar las Dificultades Especificas del Aprendizaje en Matemática. Al mismo tiempo en ellas se describe cómo afecta la Discalculia en el desarrollo del pensamiento lógico.

\section{MÉTODO}

La metodología de investigación garantiza que las relaciones que se establecen y los resultados de nuevos 
conocimientos obtenidos tengan el máximo grado de exactitud y confiabilidad. Es un procedimiento ordenado que se sigue para establecer lo significativo de los hechos y fenómenos hacia los cuales está encaminado el estudio. (Tamayo y Tamayo, 2003, p.175) En pocas palabras, es la estrategia que asume el investigador para responder al problema planteado (Arias, 2012 , p. 27). Dicho esto, en el segmento a continuación se explica la metodología que se empleó para llevar a cabo esta investigación.

Para comenzar, se puede decir el tipo de investigación fue concebido dentro de la modalidad de proyecto factible, donde cabe citar a la UPEL (2007) quien lo define como:

... la elaboración y desarrollo de una propuesta de un modelo operativo viable para solucionar problemas, requerimientos $\mathrm{o}$ necesidades de organizaciones o grupos sociales; puede referirse a la formulación de políticas, programas, tecnologías, métodos o procesos. El proyecto debe tener apoyo en una investigación de tipo documental de campo o un diseño que incluya ambas modalidades. (p.21)

Este tipo de investigaciones tienden a cumplirse por etapas generales como lo son el diagnóstico, planteamiento y fundamentación teórica de la propuesta; procedimiento metodológico, actividades y recursos necesarios para su ejecución; análisis y conclusiones sobre la viabilidad y realización del proyecto; y en caso de su desarrollo, la ejecución de la propuesta y la evaluación tanto del proceso como de sus resultados (UPEL, 2012, p. 21).
Es esa razón que la presente investigación estuvo orientada a diseñar instrumentos de evaluación para develar de manera temprana los indicadores de riesgo de las Dificultades Específicas de Aprendizaje en Matemática, dentro de la U.E.E "Juan Antonio Michelena" ubicada en el Municipio Escolar Candelaria, para intentar dar respuesta a posibles sospechas de estudiantes con Dificultades Especificas en el Aprendizaje de la Matemática.

Aunado a esto, la investigación tiene un diseño de campo no experimental, el cual consistió en la recolección de datos directamente, sin manipular o controlar variables. De forma concreta, los datos fueron recopilados en esta institución mediante la aplicación de un instrumento de recolección de datos. De acuerdo con Arias (2012) los diseños de campo son aquellos que consisten en:

...la recolección de datos
directamente de los sujetos
investigados, o de la realidad
donde ocurren los hechos (datos
primarios), sin manipular o
controlar variable alguna, es decir,
el investigador obtiene la
información, pero no altera las
condiciones existentes. De allí su
carácter de investigación no
experimental. (p.31)

A su vez, esta investigación es del tipo no experimental y transaccional descriptivo, donde los fenómenos fueron observados sin alterar, por lo cual, ocurren naturalmente en su contexto, con el fin de analizarlos luego de obtener la información acerca del problema de la investigación. Vale recalcar, que los datos fueron recopilados 
directamente de los docentes de aula en la "U.E.E. Juan Antonio Michelena".

En cuanto a la población de estudio, se le puede definir en un sentido amplio como "una parte del todo que llamamos universo y que sirve para representarlo". (Sabino, 2002, p. 83). A los fines de esta Investigación, la población estará conformada por los 24 docentes de aula de la U. E. "Juan Antonio Michelena"

En relación a la muestra, Tamayo y Tamayo (2006), al seleccionar algunos de los elementos con la intención de averiguar algo sobre la población de la cual están siendo tomados, se hace referencia a la muestra. Por lo tanto, la misma descansa en el principio de representación de las partes al todo y por tal refleja las características que definen la población. En este caso, para la muestra se seleccionaron al azar a 17 docentes de aula de $1^{\circ}$ a $6^{\circ}$ grado de las 4 secciones de cada grado, de la U.E. "Juan Antonio Michelena".

\section{RESULTADOS Y DISCUSIÓN}

Es bien conocido por todos las dificultades que poseen algunas personas para aprender matemática y aplicar esos conocimientos en su vida cotidiana, según Valencia (2015, citado por Parra, 2017), "Los problemas existentes en el aprendizaje de la asignatura de matemáticas llevan a un gran número de estudiantes al fracaso escolar" (s.n.). Dado este precedente, en el cumplimiento del primer objetivo específico referente al diagnóstico de conocimientos que poseen los docentes sobre la Discalculia, los resultados arrojaron que, aunque son pocos los profesionales que conocen el término Discalculia, la gran mayoría son capaces de reconocer los indicadores de esta discapacidad, según el estudio presentado por el instrumento de diagnóstico aplicado, el $76 \%$ de los docentes encuestados reconoce los indicadores de la Discalculia dentro del aula regular.

Sin embargo, por la naturaleza de su actividad no pueden intervenir directamente en el diagnóstico clínico de esta deficiencia, pero si puede contribuir en este proceso de corrección a través de la observación profesional de los indicadores en los escolares. Claro está, con las herramientas indicadas, ya que el docente es quien diariamente y de manera experta es testigo del desarrollo cognitivo del estudiante.

Ahora bien, dicha contribución resulta particularmente importante a la hora de disminuir el tiempo de evaluación y descubrimiento de esta dificultad específicamente, en beneficio del estudiante y su pleno desarrollo, como lo establece la LOE y la agenda 2030 de la UNESCO. Esto resulta de gran importancia pues se reducirá significativamente el tiempo invertido por los especialistas clínicos y pedagógicos en el diseño de las estrategias para atender a los educandos dentro del aula regular. En concordancia con Santana (2012), también citado por Parra (2017) "Si los conocimientos básicos en matemáticas no son dominados de forma apropiada, gran cantidad de niños, adolescentes y adultos con Discalculia tendrán dificultades al encaminarse hacia aplicaciones más complejas". Es por tanto que, como resultado del segundo objetivo específico, resulta factible la creación de instrumento para develar los indicadores de riesgo de las Dificultades Específicas del Aprendizaje de la 
Matemática en estudiantes de educación primaria ya que esto brindaría los docentes parte del apoyo que necesitan para corregir esta dificultad.

En efecto, el apoyo del docente se concentra en identificar estos indicadores dentro del aula regular, estableciendo así cuales están presentes a través del instrumento presentado a continuación, posteriormente verificar estos indicadores y por último hacer la respectiva referencia al psicopedagogo del plantel en el caso de ser una institución de educación pública (nacional, estatal o municipal) y a las unidades psicoeducativa existentes en la institución.

Por su parte, en estudios realizados por el ministerio de educación español alrededor del $25 \%$ de los alumnos con Dificultades Específicas de Aprendizaje tienen problemas con el cálculo y/o la solución de problemas, y cuando se dan combinados con problemas de lectoescritura el porcentaje aumenta hasta casi el 55\%" (Romero y Lavigne, 2005, p. 75). Así mismo, la ausencia de datos estadísticos oficiales por parte del MPPE, INE o CONAPDIS, acerca de este tipo de dificultad en la población estudiantil de Venezuela, en contraste con los resultados obtenidos, el $75 \%$ de los docentes reconocieron los indicadores de riesgo de la Discalculia, ya que coinciden con los indicadores presentes en estudiantes del aula regular, quienes seguramente no han sido diagnosticados y por ende tampoco han recibido el apoyo necesario para corregir este problema.

No cabe duda de que la ausencia de especialistas dentro de las instituciones, tanto en las públicas como en las privadas, no permite establecer la incidencia de esta dificultad específica en el aprendizaje de la matemática dentro de las aulas regulares razón por la cual el diseño de las estrategias pedagógicas necesarias por el estudiante comprometido por esta dificultad es inexistente.

Es por ello que surge la necesidad de ofrecer una herramienta que ayude al docente a determinar con mayor exactitud, si los indicadores mencionados están presentes en alguno de sus estudiantes, lo que diagnosticaría si es o no perteneciente a la población con Discalculia y de esta manera, dar inicio al proceso de diagnóstico y asesoramiento pedagógico de este estudiante. En consecuencia, resulta favorable el cumplimiento del tercer objetivo específico que propone diseñar un instrumento que permita develar los indicadores de riesgo de la Discalculia.

De allí la necesidad de formular el objetivo general de la propuesta dentro de la investigación realizada: Diseñar instrumentos que puedan ser aplicados por los docentes de aula regular a los estudiantes de educación primaria que evidencien la presencia de los indicadores de riesgo de la Discalculia. Para alcanzar este objetivo fue necesario una siguiente fase en la investigación más enfocada al diseño del instrumento en sí, para lo cual se requirió en primer lugar, Identificar cuáles son los Indicadores de riesgo de la Discalculia presentes en el aula regular, luego la verificación a través de otro instrumento de los indicadores presentes en los estudiantes de educación primaria para finalmente, referir al o los escolares de educación primaria al especialista de aula respectivo del plantel que debe estar capacitado para el asesoramiento pedagógico que requiera el educando. 
CONCLUSIONES

Se puede decir a modo de cierre que para alcanzar el desarrollo educativo en el área de matemática, se deben promover jornadas divulgativas a los docentes sobre la Discalculia y sus indicadores de riesgo, así como también, mostrar el impacto que esta dificultad tiene en la vida de las personas que resultan comprometidas por ella. Con esta jornada se garantizará que todos los docentes del plantel se capaciten en cuanto a conocimientos teóricos y metodológicos sobre la Discalculia y con la periodicidad de estas jornadas se optimizará la identificación de estos indicadores.

Como el docente de aula demostró las competencias para reconocer estos indicadores, aunado a las jornadas divulgativas programadas, se fortalecerán estas competencias. De esta manera al brindarle un instrumento de diagnóstico preciso, se le permitirá al docente de aula regular, corroborar si esos indicadores identificados luego de su observación objetiva durante el desarrollo cognitivo del estudiante en el área de matemática, representan algún riesgo comprometido con esta dificultad especifica en el aprendizaje de la matemática.

Así pues, surge la propuesta "Idear" la cual consiste en una serie de láminas proyectivas que contienen ejercicios clásicos de pruebas estandarizadas para diagnosticar la Discalculia. Dicha propuesta se encuentra dividida en 5 bloque bien definidos: Números, Operaciones, Geometría, Medidas y Probabilidad y Estadística. Durante cada bloque las láminas proyectivas de Idear estarán adaptadas al nivel de dificultad del grado correspondiente al estudiante. Estos ejercicios están basados en pruebas estandarizadas para el diagnóstico de la Discalculia

A modo de cierre, se espera que este instrumento de diagnóstico de deficiencias de la matemática colabore a la solución del bajo rendimiento académico de los estudiantes, no solo de la institución en la que se aplicó esta investigación, sino que se consolide como una herramienta pedagógica recomendada a nivel nacional, ya que la matemática es una ciencia universal que se encuentra en todas partes y de la que provienen casi todos los fundamentos para la comprensión de numerosas áreas del conocimiento y es preciso que la educación facilite su manejo y aprendizaje de manera significativa y acorde al contexto actual en el que se desenvuelve el educando.

\section{REFERENCIAS}

Arias, F. (2012). El Proyecto de Investigación. Caracas: Episteme. Recuperado de https://ebevidencia.com/wp-content/ uploads/2014/12/EL-PROYECTO-DEINVESTIGACI\%C3\%93N-6ta-Ed.-FIDIAS-G.ARIAS.pdf

Asociación Americana de Psiquiatría. (2000). Manual Diagnostico y Estadístico de los Trastornos Mentales IV. Recuperado de https://psicovalero.files.wordpress.com/20 14/06/manual-diagnc3b3stico-y-estad c3adstico-de-los-trastornos-mentalesdsm-iv.pdf[Consultado: 2019, abril 20]

Coronado A. (2012). Elaboración y validación de un instrumento de observación para detectar dificultades de aprendizaje en el cálculo aritmético. (Tesis Doctoral). Universidad de Sevilla. España Recuperado de https://idus.us.es/xmlui/bitstream/ handle/11441/70578/2012coronelabo.pdf? 
Fiuza M. y Fernández, M. (2014). Dificultades de aprendizaje y trastornos del desarrollo. Manual didáctico. (Libro Digital). Recuperado de https://mmhaler.files. wordpress.com/2012/08/fuiza-yfernandez-dificultades-de-aprendizaje-ytrastornos-del-desarrollo.pdf [consultado 2019, mayo 06]

KIRK, S. (1963): Behavioral diagnosis and remediation of lear-ning disabilities, en Procedings of the conference on exploration into the problems on the perceptually handicapped child, first annual meeting, vol. 1, Chicago

Ley Orgánica de Educación (2009). Gaceta Oficial $N^{\circ} 5.929$ Extraordinaria. Agosto 17, 2009. Caracas

Ley para Personas con Discapacidad (2007). Gaceta Oficial No 38.598. Enero de 2007. Caracas. Recuperado de http://www.tss.gob.ve/wp-content/ uploads /2016/02/LEY-PARA-LASPERSONAS-CON-DISCAPACIDAD.pdf

Llumitaxi, M. y Vera, E. (2017). La Discalculia y su incidencia en el aprendizaje de las matemáticas de los niños de 4to. Año de educación general básica de la unidad educativa "Inés maría balda" del cantón nobel de la provincia del guayas, del año lectivo 2016-2017. (Tesis de Pregrado). Universidad Laica Vicente Rocafuerte de Guayaquil, Guayaquil Ecuador, Recuperado de http://repositorio.ulvr. edu.ec/bitstream/44000/1498/1/T-ULVR1265.pdf

Méndez, S. y Vivanco, D. (2016). La descalcaría y su afectación en el proceso de desarrollo del pensamiento lógico en niños de 8 años. (Tesis de Pre-Grado). Universidad Técnica de Machala, San Antonio de Machala Provincia del Cantón Ecuador. Recuperado de http://repositorio.

utmachala.edu.ec/handle/48000/9658
Oña, L. (2016). Estudio de la Metodología de Enseñanza para Niños con Discalculia en la Escuela de Educación Básica Luis Felipe Borja. (Tesis de Pregrado). Universidad Técnica de Ambato. Ambato - Ecuador. Recuperado de http://repositorio.uta. edu.ec/bitstream/123456789/24920/1/LI GIA\%20EDITH\%200\%C3\%91A\%20CUEV A.pdf

Romero, J. y Lavigne, R. (2005). Dificultades en el Aprendizaje: Unificación de Criterios I. Definición, Características y tipo Diagnósticos. (Libro Digital) Recuperado de https://www.uma.es/ media/files/ LIBRO_I.pdf

Sabino, C. (2002). El proceso de investigación. El Cid Editor. Recuperado de

http://paginas.ufm.edu/sabino/ingles/bo ok/proceso_investigacion.pdf

Sabino, C. (2007). El Proceso de Investigación. Caracas: Panapo

Sánchez, K. (2014). La Discalculia como Trastorno de Aprendizaje de las Matemáticas en los Niños de Primer Año de Educación Básica en la Escuela "Dra. Maura Castro de Marín". Propuesta: Elaboración de recurso didáctico con herramientas multimedia. (Trabajo de Grado). Universidad de Guayaquil. Ecuador. Recuperado de http://repositorio .ug.edu.ec/handle/redug/8856? mode $=\mathrm{fu}$ II

Santamaría, G. (2014). La Evaluación de las Matemáticas en Educación Primaria. (Tesis de Pregrado). Universidad de La Rioja. La Rioja - España. Recuperado de https://biblioteca.unirioja.es/tfe_e/TFE00 0770.pdf

Tamayo y Tamayo, M. (2006). El Proceso de la Investigación Científica. Editorial Limusa. México 
UNESCO (2017). Liderar Agenda Mundial Educación 2030. Disponible en: Recuperado de https://es.unesco.org /themes/liderar-agenda-mundialeducacion-2030

Universidad Pedagógica Experimental Libertador. (2007). Manual de Trabajos de Grado de Especialización y Maestría y Tesis Doctorales de la Universidad Pedagógica Experimental Libertador. Caracas, Venezuela 